\title{
Assessment of Maternal Knowledge, Attitude and Practice towards Development of Nursing Caries among Preschool Going Children in Bangladesh
}

\author{
Wafi Ara Faruqui ${ }^{1}$ and Nadira Sultana Kakoly ${ }^{2 *}$ \\ ${ }^{1} B D S, M P H$, North South University, Dhaka, Bangladesh \\ ${ }^{2}$ Assistant Professor, Public Health Department, North South University, Dhaka, \\ Bangladesh \\ *Corresponding Author: Nadira Sultana Kakoly, Assistant Professor, Public Health \\ Department, North South University, Dhaka, Bangladesh.
}

Received: March 26, 2021

Published: August 05, 2021

(C) All rights are reserved by Wafi Ara Faruqui and Nadira Sultana Kakoly.

\section{Abstract}

Objectives: The purpose of the study was to assess the level of maternal knowledge, attitude, and practice towards the development of nursing caries among preschool-going children (2 - 5 years of age) in Bangladesh.

Methods: A cross-sectional descriptive study was conducted among 384 Bangladeshi mothers having preschool-going children (2 - 5 years of age). Using a web-based survey and a convenience sampling technique, data were collected through a self-administered questionnaire containing 34 questions on mother's knowledge of nursing caries, their family background, child's dietary habits, oral hygiene-related habits and practices, and child's feeding practices. The data were statistically analyzed using STATA version (16). The relationship between the mother's KAP and various risk factors was tested using Chi-square. Binary and multiple logistic regressions were conducted to check for independent predictors of KAP of nursing caries among pre-school-going children. A p value $<0.05$ was considered statistically significant.

Results: We found an overall good knowledge, practice, and a positive attitude score of the mothers of, 62.5\%, 70.99\%, and 53.15\% respectively. Good knowledge of mothers showed an association with their employment status ( $p$ value $=0.017$ ). However, among 384 mothers, only $36 \%$ were aware of the importance of using fluoridated toothpaste with $22 \%$ who knew the recommended portion of toothpaste to be used for 2 - 5 years old children.

Conclusion: Though maternal knowledge and attitude towards nursing caries were relatively high, it could be beneficial to create further awareness on using an appropriate amount and type of toothpaste to be used among 2 - 5-year-old children.

Keywords: Nursing Caries; Knowledge; Attitude; Practice; Preschool Children

\section{Abbreviations}

AAP: American Academy of Pediatrics; CDC: Centers for Disease Control and Prevention; HIES: Household Income and Expenditure Survey; NCD: Non-communicable Disease; WHO: World Health Organization

\section{Introduction}

Nursing Caries is a common type of tooth decay arising from specific tooth-adherent bacteria, mainly Streptococcus mutans, which metabolize acid-producing sugars demineralizing tooth structure over time [1]. It is one of the most prevalent public health 
issues in the world and is the most common non-communicable disease (NCD) among pre-school children [2]. According to the Global Burden of Disease Study in 2017, 530 million children worldwide suffer from primary dental caries [3]. Nursing caries are usually caused when a child goes to bed with milk filled bottle [4]. Moreover, babies who fall asleep while breastfeeding are also at a significantly increased risk of nursing caries. Although it is a preventable disease, if left untreated, it may cause difficulty in eating, sleeping, and may lead to severe pain and persistent systemic infection at advanced stages [2].

Though the classic etiology of Nursing Caries involves the presence of pathogenic microorganisms, dietary substrates and host must interact over a suitable period for nursing carries to occur. Numerous pieces of literature have revealed several risk factors are responsible for the development of Nursing Caries. These risk factors can be generally classified as social risk factors, including low maternal education, low socioeconomic status, lack of knowledge of oral health, and biological risk factors, including infant feeding patterns, dietary variables, early colonization of cariogenic microorganisms among others [5-7]. The American Academy of Pediatric Dentistry recommends weaning from the bottle or breastfeeding to be started from 12 - 14 months of age and discourages feeding infants during bedtime, particularly after the first tooth has emerged, to avoid the development of caries [8].

As pre-school-going children are unable to understand the importance and maintaining proper oral health care, so as primary caregivers, mostly mothers, are responsible for their daily activities. The oral health of a young child can be affected by knowledge related to oral health, the negative attitude of caregivers as well as their feeding practices [9]. Mothers with adequate oral health knowledge and actions are more likely to encourage and practice healthy oral health practices.

Globally, studies on caries among children are generally confined to children of school-going age, so a significant number of pre-school-going children are overlooked with possible nursing caries $[10,11]$. In Bangladesh, there is a scarcity of data on childhood caries for any age group of children. There is almost no evidence related to the knowledge, attitude and breastfeeding practice of Bangladeshi mothers towards the development of nursing caries among their preschool-going children. Our study will hope- fully shed more light on this topic and provide some baseline information for future work in this area. We, therefore, decided to assess the level of maternal knowledge, attitude, and practice towards the development of nursing caries among their preschool-going children in Bangladesh.

\section{Materials and Methods}

Study site and participants

A cross-sectional study was conducted from 23 September 2020 to 13 October 2020 among mothers of preschool-going children (2 - 5 years old). A total of 384 mothers participated in a web-based survey recruited through a mother's group on social media. Mothers, more than 18 years of age having children aged between 2 - 5 years old, and willing to participate were included in this study.

\section{Data collection}

A pretested and self-administered questionnaire was used for data collection. Prior informed consent was taken from the respondents and confidentiality of data was assured. The final questionnaire included questions on the following areas:

- Socio-demographic and socio-economic variables, including mother's age, education level (primary, secondary, higher secondary, university or above), mother's employment status (working/ non-working), monthly family income (which was measured according to the Bangladesh HIES-2016), place of residence (urban/ rural), child's age and sex (male/ female).

- Knowledge was measured using a 12-item questionnaire relating to general knowledge on Nursing caries and its etiology, including the presence of carious tooth in a child's oral cavity, age of onset of dental caries, the importance of regular tooth brushing, the ideal duration of tooth brushing, importance of using fluoridated toothpaste, recommended ideal portion of toothpaste for children, risk factors of dental caries, nighttime breastfeeding-related recommendation, the relationship between oral health with general health, relationship between primary teeth and permanent teeth, importance of regular dental. Responses were recorded as yes/no/don't know. The following scoring scale was used to record responses: 1 point per correct response, 0 for incorrect response or don't know. Scores varied from 0 to 12 , and a higher score suggested a good level of knowledge of mothers. 
- Attitude domain was measured using a 10-item questionnaire. Recorded items included attitudes about child's tooth cleaning, nighttime feeding, prolonged breast/ bottle feeding, dietary sugar consumption, balanced diet, regular dental checkup. The attitudes were first recorded as agree/disagree/uncertain and after scoring the recorded responses, higher scores indicated positive attitude and subsequently lower scores as negative attitude of mothers.

- Practice domains, including practice trends among mothers towards their child's oral hygiene-related behavior such as frequency and regularity of tooth brushing, method of tooth cleaning, fluoridated dentifrice use. It also included a child's feeding-related practices followed by a mother such as duration of breastfeeding, bottle feeding, nocturnal feeding practice, frequency of meal, and in-between meal snacking habit. Practices were first registered and later graded as good or poor practice to rate parameters.

\section{Data analysis}

After data collection, data were checked and verified for consistency and errors. Thereafter all data were analyzed using STATA (version 16.0). Categorical variables were described as absolute numbers and relative percentages. The associations between the outcome and the independent variables were tested using the Chisquared test with a p-value $<0.05$ considered as significant.

\section{Ethical considerations}

The study was approved by Institutional Review Board (IRB) and Ethical Review Committee (ERC) of North South University, Dhaka, Bangladesh.

\section{Results}

Socio-economic characteristics of the respondents

Out of 384 respondents, an overwhelming $97.4 \%$ were between the age group of 18 - 39 years and the remaining $2.6 \%$ were aged more than 40 years. Most of the respondents had completed university-level education (80.99\%). According to the educational status of mothers, the majority of mothers were homemakers/unemployed (67.97\%) with almost half of them (45.31\%) having a monthly family income of more than 35,000 BDT (USD 438). A vast majority of the participants (78.65\%) came from urban areas with only a fifth $(21.35 \%)$ from the rural part of the country (Table 1).

\begin{tabular}{|c|c|c|c|}
\hline Variables & Category & Frequency & Percentage \\
\hline \multirow{3}{*}{ Maternal age } & $18-29$ & 187 & $48.7 \%$ \\
\hline & $30-39$ & 187 & $48.7 \%$ \\
\hline & $>40$ & 10 & $2.6 \%$ \\
\hline \multirow{4}{*}{$\begin{array}{l}\text { Maternal } \\
\text { education level }\end{array}$} & $\begin{array}{c}\text { Primary/Elementary } \\
\text { school }\end{array}$ & 6 & $1.56 \%$ \\
\hline & \begin{tabular}{|c}
$\begin{array}{c}\text { Secondary/Middle } \\
\text { school }\end{array}$ \\
\end{tabular} & 9 & $2.34 \%$ \\
\hline & $\begin{array}{c}\text { Higher Secondary/ } \\
\text { High-School }\end{array}$ & 58 & $15.1 \%$ \\
\hline & University/Above & 311 & $80.99 \%$ \\
\hline \multirow{2}{*}{$\begin{array}{l}\text { Maternal } \\
\text { employment } \\
\text { status }\end{array}$} & Non-working & 261 & $67.97 \%$ \\
\hline & Working & 123 & $32.03 \%$ \\
\hline \multirow{4}{*}{$\begin{array}{l}\text { Monthly family } \\
\text { income (BDT) }\end{array}$} & $<15,000$ & 62 & $16.15 \%$ \\
\hline & 15,000 - 24,999 & 94 & $24.48 \%$ \\
\hline & 25,000 - 34,999 & 54 & $14.06 \%$ \\
\hline & $>35,000$ & 174 & $45.31 \%$ \\
\hline \multirow{2}{*}{$\begin{array}{l}\text { Place of } \\
\text { residence }\end{array}$} & Rural & 82 & $21.35 \%$ \\
\hline & Urban & 302 & 78.65\% \\
\hline
\end{tabular}

Table 1: Sociodemographic characteristics of the study respondents $(\mathrm{N}=384)$.

Maternal knowledge on nursing caries during early childhood

The overall knowledge score of mothers was relatively high with $62.5 \%$ of mothers having good overall knowledge. The percentage of responses from the knowledge domain is shown in detail in figure 1. Most of the study participants had above-average knowledge of the presence or absence of carious teeth in their child's oral cavity (97.92\%) as well as on the importance of tooth brushing in the prevention of tooth decay (98.1\%). 86.98\% and $84.37 \%$ of respondents recognized dental caries as a disease and sugar/sugary food to be a risk factor for the development of dental caries. $72.66 \%$ and $64.58 \%$ of respondents claimed that oral health can eventually affect the general systemic health of the child. They mentioned in 
particular that dental caries in primary teeth can affect the eruption of permanent teeth. A slightly fewer number $(64.32 \%)$ and $(61.2 \%)$ of mothers knew the age of onset of dental caries and the ideal duration of tooth brushing.

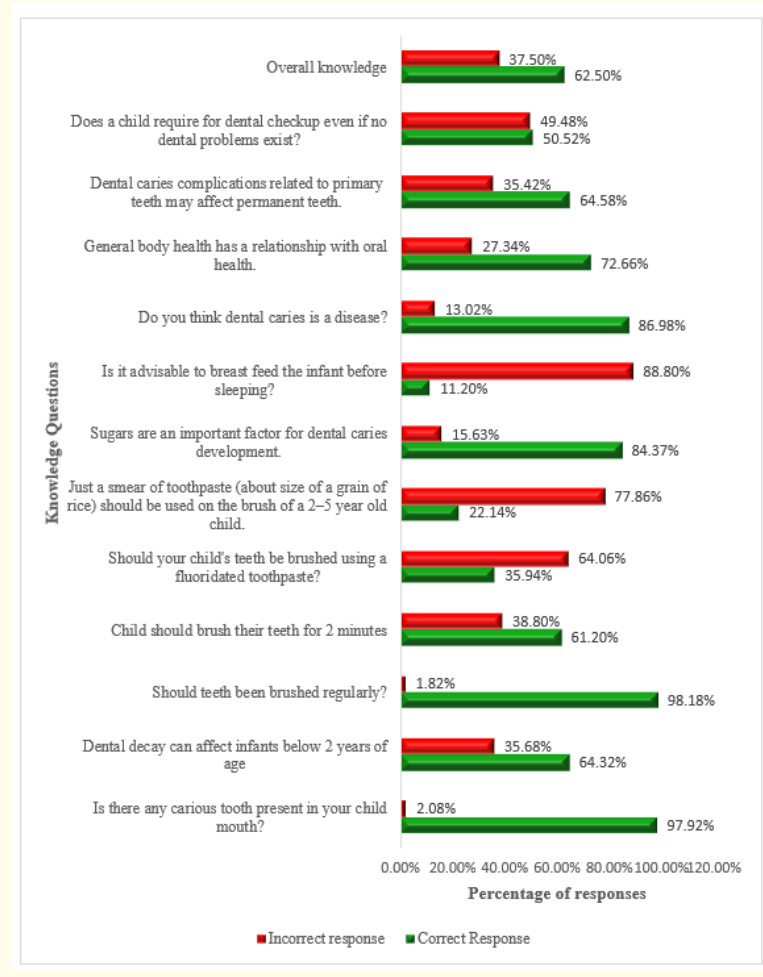

Figure 1: Percentage of mothers with correct knowledge related to nursing caries.

In contrast, only about $11.2 \%$ of mothers reported that nocturnal breastfeeding may be detrimental. Only $35.94 \%$ of respondents were aware of the importance of using fluoridated toothpaste for their children, with $78 \%$ of the mothers being uninformed of the ideal portion of toothpaste that a child should use.

\section{Maternal attitude towards oral hygiene}

An overall positive attitude was seen among mothers towards the oral hygiene of their pre-school-going children. Most of the participants (95.57\%) admitted that a balanced diet is necessary for the development of healthy gums and teeth with nearly $90 \%$ of the mothers displaying a positive attitude about visiting a dentist at regular intervals for their children. Almost $84.64 \%$ of the respon- dents, also believed that milk teeth should be taken good care of and should be cleaned by mothers or with her assistance.

About three-quarters of the respondents $(76.04 \%$ and $75.26 \%$ respectively) indicated that the cleaning of a child's teeth should be initiated from the very beginning of eruption of the first baby tooth and before the eruption of the first tooth, in particular, that the mouth should be cleaned with gauge. Almost $65.89 \%$ of participants stated that a child's mouth should be cleaned after every meal.

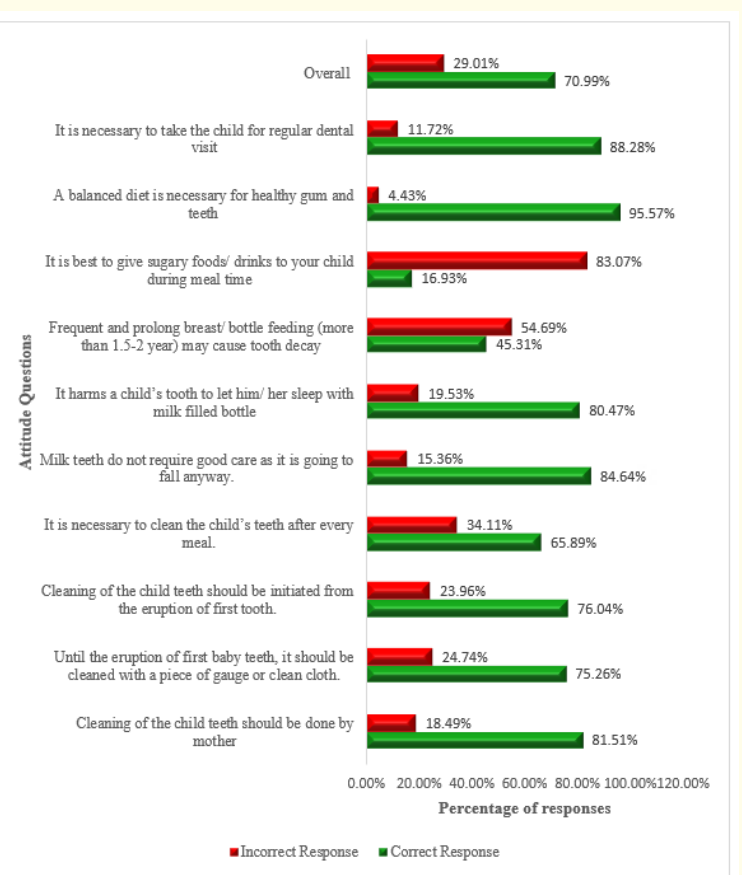

Figure 2: Maternal responses for attitude domain.

\section{Oral hygiene practice}

The percentage of responses from practice domain questions is shown in figure 3 . The overall good practice score of the respondents in this study was $53.15 \%$ which indicates a gap in the child's oral hygiene and associated feeding behavior. According to the study participants, $67.61 \%$ of children brushed their teeth regularly and most of them ( $94.27 \%$ and $95.05 \%$ respectively) used toothbrushes and dentifrices to clean their teeth. However, just $30.68 \%$ of the respondents reported using fluoridated dentifrices on their children, while only $23.18 \%$ reported cleaning their child's teeth 
Assessment of Maternal Knowledge, Attitude and Practice towards Development of Nursing Caries among Preschool Going Children in Bangladesh

twice a day. Only a third (30.47\%) of mothers claimed to have ever visited a dentist with their child.

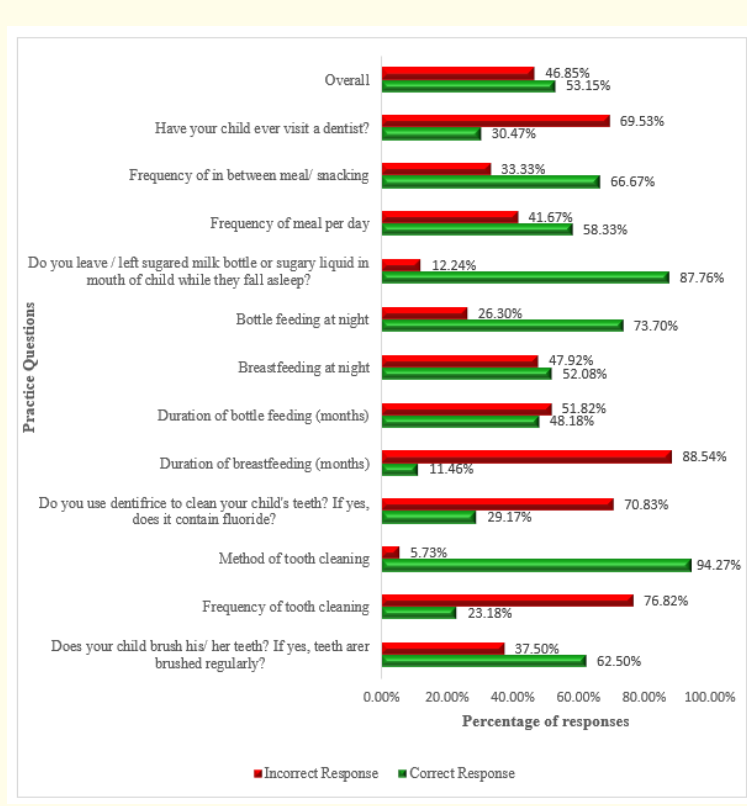

Figure 3: Percentage of responses for practice questions.
While almost half of the participants (48.18\%) demonstrated proper bottle-feeding practice, only $11.46 \%$ showed proper breastfeeding practice for their children. More than half of the respondents $(73.7 \%$ and $52.08 \%$ respectively) didn't practice nocturnal bottle and breastfeeding but practiced snacking in between meals (58.33\% and $66.67 \%$ respectively).

Factors associated with knowledge, attitude, and practice of mothers related to nursing caries

Among the socio-demographic variables, this study only found a statistically significant association between the good knowledge of mothers with their employment status. It was quite surprising to note that, non-working mothers showed comparatively higher levels of knowledge compared to those who were working (Table 2 ). This could be because children of working mothers are usually taken care of by other caregivers who would most likely have had better knowledge of the actual practice of the child.

Factors associated with self-reported caries

Table 3 illustrates that there was no relationship between children's feeding practice and presence of self-reported caries rather

\begin{tabular}{|c|c|c|c|c|c|}
\hline & & \multirow{3}{*}{ Total } & \multicolumn{2}{|c|}{ Knowledge } & \multirow[b]{3}{*}{ P-value } \\
\hline \multirow{2}{*}{\multicolumn{2}{|c|}{ Variables }} & & Good & Poor & \\
\hline & & & F (\%) & F (\%) & \\
\hline \multirow[b]{3}{*}{ Mother's age group } & $18-29$ & 187 & $128(48.30 \%)$ & $59(49.58 \%)$ & \multirow{3}{*}{$\begin{array}{c}0.973 \\
\text { Fisher's exact }=0.976\end{array}$} \\
\hline & $30-39$ & 187 & $130(49.06 \%)$ & $57(47.9 \%)$ & \\
\hline & $>40$ & 10 & $7(2.64 \%)$ & $3(2.52 \%)$ & \\
\hline \multirow{4}{*}{ Mother's education level } & Primary & 6 & $5(1.89 \%)$ & $1(0.84 \%)$ & \multirow{4}{*}{ Fisher's exact $=0.708$} \\
\hline & Secondary & 9 & $5(1.89 \%)$ & $4(3.36 \%)$ & \\
\hline & Higher Secondary & 58 & $39(14.72 \%)$ & $19(15.97 \%)$ & \\
\hline & University/above & 311 & $216(81.50 \%)$ & $95(79.83 \%)$ & \\
\hline \multirow{2}{*}{$\begin{array}{l}\text { Mother's employment } \\
\text { status }\end{array}$} & Non-working & 261 & $170(64.15 \%)$ & $91(76.47 \%)$ & \multirow{2}{*}{0.017} \\
\hline & Working & 123 & $95(35.85 \%)$ & $28(23.53 \%)$ & \\
\hline \multirow{4}{*}{ Monthly family income } & $<15,000 \mathrm{BDT}$ & 62 & $43(16.23 \%)$ & $19(15.97 \%)$ & \multirow{4}{*}{0.761} \\
\hline & 15,000 - 24,999 BDT & 94 & $61(23.02 \%)$ & $33(27.73 \%)$ & \\
\hline & 25,000 - 34,999 BDT & 54 & $37(13.96 \%)$ & $17(14.28 \%)$ & \\
\hline & $>35,000$ BDT & 174 & $124(46.79 \%)$ & $50(42.02 \%)$ & \\
\hline \multirow[t]{2}{*}{ Place of residence } & Rural & 82 & $57(21.51 \%)$ & $25(21 \%)$ & \multirow{2}{*}{0.912} \\
\hline & Urban & 302 & $208(78.49 \%)$ & $94(79 \%)$ & \\
\hline
\end{tabular}

Table 2: Association of knowledge of mothers with different variables $(\mathrm{N}=384)$. 
Assessment of Maternal Knowledge, Attitude and Practice towards Development of Nursing Caries among Preschool Going Children in Bangladesh

it was the child's regular tooth brushing practice (p-value $<0.05)$, presence of Fluoride in their dentifrices ( $p$ value $<0.05$ ) and using an appropriate portion of toothpaste for tooth cleaning ( $\mathrm{p}$ value $<0.05$ ) which was associated with self-reported caries in these young children. Our study found that more than $60 \%$ of mothers didn't know whether their children's toothpaste contains Fluoride or not nor how much toothpaste should be used for a 2 - 5 years old child.

\begin{tabular}{|c|c|c|c|c|}
\hline \multirow{2}{*}{\multicolumn{2}{|c|}{ Variables }} & \multicolumn{2}{|c|}{ Presence of Caries } & \multirow{2}{*}{ p-value } \\
\hline & & & Absent & \\
\hline Duration of breastfeeding (months) & 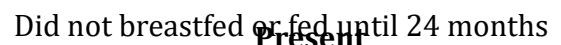 & $57(57 \%)$ & $43(43 \%)$ & 0.661 \\
\hline \multirow{2}{*}{ Duration of bottle feeding (months) } & Did not bottle-fed or fed until 24 months & $168(57.53 \%)$ & $124(42.47 \%)$ & \multirow{2}{*}{0.349} \\
\hline & Bottle-fed $>24$ months & $58(63.04 \%)$ & $34(36.96 \%)$ & \\
\hline Regular tooth brushing practice & No & $78(67.83 \%)$ & $37(32.17 \%)$ & 0.012 \\
\hline \multirow{3}{*}{ Presence of Fluoride in dentifrice } & Yes & $73(65.18 \%)$ & $39(34.82 \%)$ & \multirow{3}{*}{0.007} \\
\hline & No & $22(40.74 \%)$ & $32(59.26 \%)$ & \\
\hline & Don't know & $124(62.31 \%)$ & $75(37.69 \%)$ & \\
\hline
\end{tabular}

Table 3: Relationship between the presence of caries and different variables.

\section{Discussion}

Nursing caries is becoming increasingly common among preschool-going children all over the world and is now becoming a public health concern. In the long term, poor oral health can impact general systemic health as oral health is a gateway to systemic disease. However, the presence of caries among this very young age group remains mostly neglected by public health experts globally. One of the ways to improve this situation would be to increase primary caregivers' knowledge related to proper oral health care which will hopefully lead to better practices. A good practice that is developed from the very early stage of life later on become reinforcing in nature and resistant to change.

Overall knowledge, attitude, and practice of mothers

According to our findings, almost $62.5 \%$ of mothers had good knowledge of nursing caries, $71.5 \%$ expressed a positive attitude and only 53\% exercised good practices regarding their child's oral hygiene and feeding-related behavior. Our findings are in agreement with a study conducted by Sogi., et al. 2020, assessing the
Knowledge, attitude, and practices of oral health care in the prevention of early childhood caries among parents of children in Belagavi city who also found a similar mean knowledge score of $69.5 \%$ [5].

Toothpaste use

In our study, only a third (35.94\%) of mothers were aware of using fluoridated toothpaste for their children. This is significantly lower than other caregivers among Moroccan mothers as seen in a study conducted by Chala., et al. 2018, where almost $60.9 \%$ of mothers were aware of the use of fluoridated toothpaste for their children [12]. Similarly, in our study, only a fifth of our respondent (22.14\%) mothers had knowledge of the ideal portion of toothpaste recommended for use in 2 - 5-year-old children which was substantially lower than that found among Saudi Arabian mothers [13]. Our data indicate a considerable knowledge gap among Bangladeshi mothers in particular about the ideal portion of toothpaste use as well as the importance of fluoride in it, which could be our areas of further focus. 
Breastfeeding and bottle feeding

From this research study, we have found that respondents showed a positive attitude about the harmful effects that nocturnal feeding $(80.47 \%)$ had on caries but displayed a negative attitude towards the harmful impact of prolonged breast/bottle feeding (45.31\%). Sogi., et al. had similarly observed that only $45.5 \%$ of parents assumed that nocturnal feeding was detrimental, but in contrast to our study findings, he observed that prolonged and frequent breastfeeding could also be harmful [5]. Our study indicates that Bangladeshi mothers do not view prolonged breast/ bottle feeding as detrimental to or related to dental caries development. About 73.96\% of Bangladeshi mothers breastfed their child for more than 24 months which is contradictory to WHO, AAP, and CDC guidelines for breastfeeding [14-16]. Our findings indicate the need for further health education along these areas.

The study also revealed that there was a statistically significant $(p<0.05)$ relationship between the presence of caries with regular tooth brushing practice, use of fluoridated dentifrice as well as the use of an ideal portion of toothpaste. Children who did not brush their teeth regularly, had a higher prevalence of caries, while mothers who did not know about the ideal portion of toothpaste that should be used in children as well as the chemical constituents of the toothpaste, in particular, whether it contained fluoride, reported a higher presence of carious tooth in their child's oral cavity. While a vast majority of our respondent mothers were educated up to university level, a clear gap in knowledge regarding this indicates areas for further research in larger population-based studies to validate our findings.

As with all online surveys, a limitation of our study was that it was not possible to conduct an intraoral examination such that the self-reported responses of mothers may not reflect actual behavior. However, it is assumed that a carious tooth with its symptoms and visibility would be clear to a primary caregiver reducing likely misclassifications.

\section{Conclusion}

The result of this study reveals that despite having good knowledge and a positive attitude, many mothers did not practice the same in their daily lives. Mothers are known to be the prime caregivers of a child and play a very important part in fostering healthy oral behavior. From this research, we have identified a knowledge and practice gap among mothers concerning nocturnal feeding habits and regular teeth brushing, as well as the use of fluoridated toothpaste.

Regular oral health education and promotion activities should be conducted, emphasizing the importance of choosing fluoridated toothpaste, using the ideal portion of toothpaste as well as on infant feeding habits. More commercial advertisements on childspecific toothpaste should be created to promote its use and its benefits. According to ADA recommendation, children younger than 3 years should use Fluoride toothpaste which is no more than a smear or the size of a grain of rice. Excessive or inadequate intakes may lead to dental fluorosis which can ultimately weaken the teeth by hampering the development of permanent teeth [19]. In addition, mothers should be trained on effective breast and bottle feeding practices.

\section{Conflict of Interest}

The authors have no conflict of interest to declare.

\section{Acknowledgment}

We would like to thank all the mothers who participated in this research and spent their precious time filling out the questionnaire. We would also like to thank Dr. Shah Mahmud Mishu and Nandeeta Samad for their support and valuable suggestions during the study. We would also like to thank the North-South University Ethics Review Committee for permitting us to perform this study.

\section{Bibliography}

1. Rathee M and Sapra A. "Dental Caries". In: StatPearls. Treasure Island (FL): StatPearls (2020).

2. World Health Organization. Sugars and Dental Caries (2017).

3. WHO. Oral health (2020).

4. Tungare S and Paranjpe AG. "Baby Bottle Syndrome (2020).

5. Sogi H., et al. "Knowledge, attitude, and practices of oral health care in prevention of early childhood caries among parents of children in Belagavi city: A Questionnaire study". Journal of Family Medicine and Primary Care 5.2 (2016): 286-290.

6. Togoo R., et al. "Caregivers' Knowledge, Attitudes, and Oral Health Practices for Infants Attending Day-care Centers in 
two cities in Southern Saudi Arabia". Gulf Medical Journal 6.1

(2017): 35-41.

7. Vakani F., et al. "Oral Hygiene KAP Assessment and DMFT scoring among children". Journal of College of Physicians and Surgeons Pakistan 21.4 (2011): 223-226.

8. Nainar SMH and Mohummed S. "Diet counseling during the infant oral health visit". Pediatric Dentistry 26.5 (2004): 459-462.

9. American Academy of Pediatric Dentistry. "Perinatal and infant oral health care. The Reference Manual of Pediatric Dentistry". Chicago, Ill.: American Academy of Pediatric Dentistry (2020): 252-256.

10. Bangladesh Demographic Health Survey, 2017-2018.

11. Progotir Pathey Bangladesh. Multiple Indicator Cluster Survey (2019).

12. Chala S., et al. "Knowledge, attitudes and self-reported practices toward children oral health among mother's attending maternal and child's units, Salé, Morocco". BMC Public Health 18 (2018): 618.

13. G RK., et al. "Parent's Knowledge, Attitude and Practice on Prevention of Early Childhood Caries in Al jouf Province, Saudi Arabia”. Pesquisa Brasileira em Odontopediatria e Clínica Integrada 18.1 (2018): e3837.

14. American Academy of Pediatrics. Breastfeeding and the use of human milk". Pediatrics 129.3 (2012): e827-e841.

15. World Health Organization. The World Health Organization's infant feeding recommendation. (2001).

16. Centers for Disease Control and Prevention. U.S. Breastfeeding Rates Are Up! More Work is Needed (2017).

17. Khanduri N., et al. "Knowledge, attitude, and practices of parents toward their children's oral health: A questionnaire survey in Bhairahawa (Nepal)". International Journal of Pedodontic Rehabilitation 3 (2018): 59-61.

18. D Nivedha., et al. "Association of knowledge and attitude of mothers and pre-nursery / nursery school teachers regarding early childhood caries in preschool children of Puducherry - a cross sectional study". International Journal of Contemporary Medical Research 4.3 (2017): 676-680.

19. American Dental Association Council on Scientific Affairs. "Fluoride toothpaste use for young children". The Journal of the American Dental Association 145.2 (2014): 190-191.

\section{Volume 5 Issue 9 September 2021 \\ (C) All rights are reserved by Wafi Ara Faruqui and Nadira Sultana Kakoly.}

